\title{
COMPUTATION OF RAMSEY NUMBERS BY P SYSTEMS WITH ACTIVE MEMBRANES
}

\author{
LINQIANG PAN \\ Image Processing and Intelligent Control Key Laboratory of Education Ministry \\ Department of Control Science and Engineering \\ Huazhong University of Science and Technology \\ Wuhan 430074, Hubei, China \\ lqpan@mail.hust.edu.cn \\ DANIEL DÍAZ-PERNIL and MARIO J. PÉREZ-JIMÉNEZ \\ Department of Computer Science and Artificial Intelligence, University of Sevilla Avda. \\ Reina Mercedes s/n, 41012 Sevilla, Spain \\ sbdani@us.es \\ marper@us.es
}

\begin{abstract}
Ramsey numbers deal with conditions when a combinatorial object necessarily contains some smaller given objects. It is well known that it is very difficult to obtain the values of Ramsey numbers. In this work, a theoretical chemical/biological solution is presented in terms of membrane computing for the decision version of Ramsey number problem, that is, to decide whether an integer $n$ is the value of Ramsey number $R(k, l)$, where $k$ and $l$ are integers.
\end{abstract}

Keywords: Membrane computing; P system; Ramsey number.

\section{Introduction}

In 1930 the British mathematician Frank P. Ramsey published a paper that has led to a research area called Ramsey Theory [8]. Ramsey-type theorems have roots in different branches of mathematics and the theory developed from them influenced such diverse areas as number theory, set theory, geometry, ergodic theory and theoretical computer science [9]. Ramsey-type theorems are showing that a total disorder is impossible. Specifically, Ramsey theory studies conditions when a combinatorial object necessarily contains some smaller given objects. The role of Ramsey numbers is to quantify some of the general existential theorems in Ramsey theory.

For integers $k, l \geq 2$, the classical 2-color Ramsey number $R(k, l)$ is defined as the least number $n=n(k, l)$ such that for every 2-coloring (say red and green) of the edges of the complete graph $K_{n}$ on $n$ vertices, $K_{n}$ contains either a clique of 
order $k$ with red edges or a clique of order $l$ with green edges. It is well known that the clique problem is an NP-complete problem [2]. Furthermore, computing a lower bound $L$ for $R(k, l)$ usually requires exhibiting a red/green coloring of the complete graph $K_{L-1}$ with no red $K_{k}$ subgraph and no green $K_{l}$ subgraph. Searching all colorings of a graph $K_{L-1}$ becomes computationally extremely difficult as $L$ increases; the number of colorings grows super-exponentially. So it is not easy to obtain the exact values of Ramsey numbers. The difficulty of computing Ramsey numbers was summed up quite nicely by the great Hungarian mathematician, Paul Erdös: Suppose an evil spirit would tell us, "Unless you tell me the value of $R(5,5)$ I will exterminate the human race." Our best strategy would perhaps be to get all the computers and computer scientists to work on it. If he would ask for $R(6,6)$ our best bet would perhaps be to try to destroy him before he destroys us [1].

Until now, there are only nine known nontrivial values for Ramsey numbers $R(k, l)$ [7], see Table 1 . It is a great challenge for mathematician and computer scientists to compute the exact values of Ramsey numbers. In this work, a new way is given for computing Ramsey numbers based on a kind of "chemical/biological" computational devices, instead of physical computational devices.

Table 1. Known nontrivial values for $R(k, l)$.

\begin{tabular}{crrrrrrrrr}
\hline$k$ & 3 & 3 & 3 & 3 & 3 & 3 & 3 & 4 & 4 \\
$l$ & 3 & 4 & 5 & 6 & 7 & 8 & 9 & 4 & 5 \\
$R(k, l)$ & 6 & 9 & 14 & 18 & 23 & 28 & 36 & 18 & 25 \\
\hline
\end{tabular}

Membrane systems (usually called $P$ systems) are a class of distributed parallel computing devices of a biochemical type, introduced in [3], which can be seen as a general computing architecture where various types of objects can be processed by various operations. The idea originates from the observation that certain processes which take place in the complex structure of living organisms can be considered as computations. We refer the reader to [5] for a motivation and detailed description of various $\mathrm{P}$ system models. In this work, $P$ systems with active membranes are considered [4]. For a quick overview of $\mathrm{P}$ systems with active membranes, we refer the reader to Chapter 11 in [6].

In this work, an algorithm is presented in terms of $\mathrm{P}$ systems with active membranes to decide whether an integer number $n$ is the value of Ramsey number $R(k, l)$, whose computation time is polynomial with respect to $n$. If a lower bound $L$ and an upper bound $U$ of a Ramsey number $R(k, l)$ are known and the gap $U-L$ between the lower bound and upper bound is not big, then the value of Ramsey number $R(k, l)$ can be obtained by running the above algorithm at most $U-L$ times. For example, $43 \leq R(5,5) \leq 49$, so it needs only to run the above algorithm at most 6 times in order to get the exact value of $R(5,5)$. Hence, the algorithm given in this work looks attractive. 


\section{Computing Ramsey Numbers by P Systems}

Ramsey numbers $R(k, l)(k, l \geq 2)$ can be considered as a function from $\mathbb{N}^{2}$ to $\mathbb{N}$. The decision version of Ramsey number problem can be expressed as follows: given integers $n, k, l$, decide whether or not $n$ is the value of Ramsey number $R(k, l)$.

The resolution of Ramsey number problem given below is a brute force algorithm, which consists of the following stages:

- Generation Stage (I): Using membrane division for non-elementary membranes, all complete graphs $K_{n}$ with different edge 2-colorings (say red and green) are produced.

- Generation Stage (II): For each complete graph $K_{n}$ with edges colored by red or green, all subsets of vertices are generated by using membrane division for non-elementary membranes.

- Checking Stage (I): For each complete graph $K_{n}$ with edges colored by red or green, we check whether there is a clique with $k$ vertices and red edges.

- Checking Stage (II): For each complete graph $K_{n}$ with edges colored by red or green, if there is no clique with $k$ vertices and red edges, then we check whether there is a clique with $l$ vertices and green edges.

- Output Stage: The system sends to the environment the right answer according to the results of the previous stage. If in all complete graphs $K_{n}$ with edges colored by red or green, there is a clique with $k$ vertices and red edges or a clique with $l$ vertices and green edges, then the answer is yes. Otherwise, the answer is no.

Let us consider the (polynomial time computable and bijective) function $\langle n, k, l\rangle$ from $\mathbb{N}^{3}$ onto $\mathbb{N}$, where $\langle n, k, l\rangle=\langle n,\langle k, l\rangle\rangle$, induced by $\langle x, y\rangle=((x+l)(x+$ $y+1) / 2)+x$. For given $n, k, l$, a $\mathrm{P}$ system with active membranes $\Pi(\langle n, k, l\rangle)$ is constructed to decide whether $n$ is the value of Ramsey number $R(k, l)$.

$$
\begin{aligned}
& \Pi(\langle n, k, l\rangle)=\left(O(\langle n, k, l\rangle), H, \mu, w_{0}, w_{1}, w_{2}, w_{3}, w_{4}, R\right), \text { where } \\
& O(\langle n, k, l\rangle)=\left\{a_{i}, t_{i}, f_{i}, \bar{t}_{i}, \bar{f}_{i}, f_{i}^{\prime}, \bar{f}_{i}^{\prime}, y_{i}, z_{i}, \bar{y}_{i}, \bar{z}_{i} \mid 1 \leq i \leq n\right\} \cup\left\{g_{i} \mid 0 \leq i \leq n+3\right\} \\
& \cup\left\{d_{i} \mid 1 \leq i \leq n(n-1)+3\right\} \cup\left\{h_{i}, h_{i}^{\prime} \mid 0 \leq i \leq \max \{k, l\}\right\} \cup\left\{a_{i, j} \mid \leq i<j \leq n\right\} \\
& \cup\left\{e_{i, j, s} \mid 1 \leq i \leq n+2,1 \leq j \leq n+2,0 \leq s \leq 2 l+7\right\} \\
& \cup\left\{\bar{e}_{i, j, s} \mid 1 \leq i \leq n+2,1 \leq j \leq n+2,0 \leq s \leq 2 n+2 k+6\right\} \\
& \cup\left\{c_{i} \mid 0 \leq i \leq n^{2}+5 n+2 k+2 l+26\right\} \cup\left\{a, b, g, h, p, q, u, \text { yes, no, no }{ }_{1}, \text { no }_{2}, \text { no }_{3}\right\},
\end{aligned}
$$

(1) $\left[a_{i, j}\right]_{4}^{0} \rightarrow\left[e_{i, j, 2 l+7}\right]_{4}^{+}\left[\bar{e}_{i, j, 2 n+2 k+6}\right]_{4}^{-}, 1 \leq i<j \leq n$.

(2) $\left[[]_{i+1}^{+}[]_{i+1}^{-}\right]_{i}^{0} \rightarrow\left[[]_{i+1}^{0}\right]_{i}^{+}\left[[]_{i+1}^{0}\right]_{i}^{-}, i=2,3 . \quad(3)\left[[]_{2}^{+}[]_{2}^{-}\right]_{1}^{0} \rightarrow\left[[]_{2}^{0}\right]_{1}^{0}\left[[]_{2}^{0}\right]_{1}^{0}$.

The objects $a_{i, j}$ corresponds to edges $\left(v_{i}, v_{j}\right), 1 \leq i<j \leq n$. Using the rules of type (1), for non-deterministically chosen $a_{i, j}$, two objects $e_{i, j, 2 l+7}$ and $\bar{e}_{i, j, 2 n+2 k+6}$ are produced, placed in two separate copies of membrane 4 , 
where $e_{i, j, 2 l+7}$ denotes that edge $\left(v_{i}, v_{j}\right)$ is colored with red, and $\bar{e}_{i, j, 2 n+2 k+6}$ denotes that edge $\left(v_{i}, v_{j}\right)$ is colored with green. The rules of types (2) and (3) are division rules for membrane with label 1, 2 and 3 , to be used in steps which follow the use of the rules of type (1). The division of a membrane with label 4 is propagated from lower levels to upper levels of the membrane structure and the membranes are continuously divided. The membrane division stops at the level where the membrane has the label 1. During the division, the charge of the membranes with label 2, 3 and 4 first changes to positive or negative, then returns to neutral, hence the process can continue. In this way, in $n(n-1)+2$ steps all $2^{n(n-1) / 2}$ graphs with different coloring of edges are generated, placed in $2^{n(n-1) / 2}$ separate copies of membrane 4 . The structure of the system after $n(n-1)+2$ steps is shown in Figure 1 .

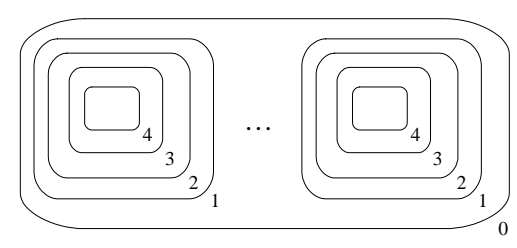

Fig. 1. The structure of system after step $n(n-1)+2$.

(4) $\left[d_{i} \rightarrow d_{i+1}\right]_{4}^{\alpha}, \alpha \in\{+,-, 0\}, 1 \leq i \leq n(n-1)+2$. (5) $\left[d_{n(n-1)+3}\right]_{4}^{0} \rightarrow d_{1}$. At step $n(n-1)+3$, the counter $d_{n(n-1)+3}$ dissolves the membrane with label 4 , and change to object $d_{1}$. The object $d_{1}$ is used as a counter again in membranes with label 3 . The computation passes to the next stage - for each graph with different coloring of edges, all subsets of vertices will be generated in order to check whether the corresponding graph has a clique $K_{k}$ with red edges or a clique $K_{l}$ with green edges.

(6) $\left[a_{i}\right]_{3}^{0} \rightarrow\left[t_{i}\right]_{3}^{+}\left[f_{i}\right]_{3}^{+}, 1 \leq i \leq n . \quad(7)\left[[]_{3}^{+}[]_{3}^{-}\right]_{2}^{0} \rightarrow\left[[]_{3}^{0}\right]_{2}^{0}\left[[]_{3}^{0}\right]_{2}^{0}$.

The objects $a_{i}$ correspond to vertices $v_{i}, 1 \leq i \leq n$. Using the rules of type (6), for non-deterministically chosen $a_{i}$, two objects $t_{i}$ and $f_{i}$ are produced, placed in two separate copies of membrane 3 , where $t_{i}$ denotes that vertex $v_{i}$ appears in some subset of vertices, and $f_{i}$ denotes that vertex $v_{i}$ does not appear in some subset of vertices. The division rules of type (7) returns the charge of membranes with label 3 to neutral, so the process can continue. In $2 n$ steps, all $2^{n}$ subsets of the vertex set $V$ are generated for each graph. After step $n^{2}+n+3$, the structure of the system is shown in Figure 2 .

(8) $\left[d_{i} \rightarrow d_{i+1}\right]_{3}^{0}, 1 \leq i \leq n-1$. (9) $\left[d_{n} \rightarrow q q h_{0}\right]_{3}^{0}$.

At step $n^{2}+n$, the subscript of the counter $d_{i}$ reaches $n-1$, and at step $n^{2}+n+2$, objects $q$ and $h_{0}$ are introduced; the objects $q$ will exit the membrane (at steps $n^{2}+n+4, n^{2}+n+5$ ), changing its polarization; the object $h_{0}$ is a new counter which will be used at the subsequent steps as shown below. 
The next stage of the computation now starts - counting the number of objects $t_{i}(1 \leq i \leq n)$ in each membrane with label 3 , which corresponds to the cardinality of each subset; the subsets with cardinality exactly $k$ will be selected out.

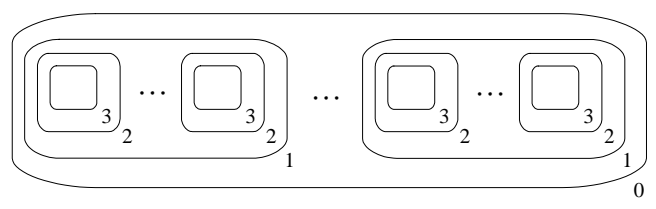

Fig. 2. The structure of system after step $n^{2}+n+3$.

(10)
$[q]_{3}^{0} \rightarrow[]_{3}^{-} u$.
(11) $[q]_{3}^{-} \rightarrow[]_{3}^{0} u$.
(12) $\left[t_{i} \rightarrow \bar{t}_{i} a b\right]_{3}^{-}, 1 \leq i \leq n$.
(13) $\left[f_{i} \rightarrow \bar{f}_{i} f_{i}^{\prime}\right]_{3}^{-}, 1 \leq i \leq n$.
(14) $\left[h_{i} \rightarrow h_{i}^{\prime}\right]_{3}^{0}, 0 \leq i \leq k$.
(15) $\left[h_{i}^{\prime} \rightarrow h_{i+1}\right]_{3}^{+}, 0 \leq i \leq k-1$.
(16) $[a]_{3}^{0} \rightarrow[]_{3}^{+} u$.
(17) $[b]_{3}^{+} \rightarrow[]_{3}^{0} u$.

At step $n^{2}+n+4, h_{0}$ evolves to $h_{0}^{\prime}$, and at the same time one copy of $q$ exits the membrane, changing its polarization to negative, where the object $u$ is a dummy object that will never evolve again. At step $n^{2}+n+5, t_{i}$ evolves to $\bar{t}_{i} a b, f_{i}$ evolves to $\bar{f}_{i} f_{i}^{\prime}$, and at the same time the other copy of $q$ exits the membrane, changing its polarization to neutral. The objects $\bar{t}_{i}$ and $\bar{f}_{i}$ have the same meaning as objects $t_{i}$ and $f_{i}$ : the vertex $v_{i}$ appear or doesn't appear in some subset of vertices, respectively; they will be used in checking whether there is a copy of clique $k_{l}$ with red edges or green edges (the process will take place in membranes with label 2 as you will see below). At step $n^{2}+n+6$, one copy of $a$ exits the membrane, changing its polarization to positive. At step $n^{2}+n+7, h_{0}^{\prime}$ evolves to $h_{1}$, at the same time one copy of $b$ exits the membrane, returning its polarization to neutral (this makes possible the use of rules of types (14) and (16)).

The rules of types (14) - (17) are applied as many times as possible. Clearly, at step $n^{2}+n+5+2 k$, a membrane contains object $h_{k}$ if and only if the cardinality of the corresponding subset is at least $k$. At step $n^{2}+n+6+2 k$, in the membrane whose corresponding subset has cardinality more than $k$, $h_{k}$ evolves to $h_{k}^{\prime}$, and one copy of $a$ changes its polarization to positive. This membrane will no longer evolve, as no further rule can be applied to it. In the membrane whose corresponding subset has cardinality exactly $k, h_{k}$ evolves to $h_{k}^{\prime}$, and its polarization remains neutral, because there is no copy of $a$ which can be used. The next stage of computation starts- checking whether a subset with cardinality $k$ is a clique.

(18)

$\left[h_{k}^{\prime} \rightarrow q q g\right]_{3}^{0} . \quad(19)\left[f_{i}^{\prime} \rightarrow y_{i} z_{i}\right]_{3}^{-}, 1 \leq i \leq n . \quad$ (20) $\left[g \rightarrow g_{0}\right]_{3}^{-}$.

(21) $\left[\bar{e}_{i, j, r} \rightarrow \bar{e}_{i, j, r-1}\right]_{3}^{\alpha}, 1 \leq i<j \leq n, 1 \leq r \leq 2 n+2 k+6, \alpha \in\{+,-, 0\}$.

At step $n^{2}+n+7+2 k$, in the membranes with label 3 and polarization 0 , $h_{k}^{\prime}$ evolves to $q q g$. At step $n^{2}+n+8+2 k$, one copy of $q$ exits the membrane, changing its polarization to negative. At step $n^{2}+n+9+2 k, f_{i}(1 \leq i \leq n)$ 
evolves to $y_{i} z_{i}, g$ evolves to $g_{0}$, and an object $\bar{e}_{i, j, 0}$ appears for each edge $\left(v_{i}, v_{j}\right)$ with green color (note that the objects $\bar{e}_{i, j, 2 n+2 k+6}$ are released from membranes with label 4 to membranes with label 3 at step $n(n-1)+3$ by using the rule (5); from that time, the rules of type (21) start to work). At same time, the other copy of $q$ exits the membrane, changing its polarization to neutral.

(22) $\left[y_{i} \rightarrow y_{i+1}\right]_{3}^{0}, 1 \leq i \leq n-1 . \quad(23)\left[z_{i} \rightarrow z_{i+1}\right]_{3}^{0}, 1 \leq i \leq n-1$.

(24) $\left[\bar{e}_{i, j, 0} \rightarrow \bar{e}_{s(i), s(j), 0}\right]_{3}^{0}, 1 \leq i<j \leq n+1$, where $s(t)=\min (t+1, n+2)$.

(25) $\left[g_{i} \rightarrow g_{i+1}\right]_{3}^{0}, 1 \leq i \leq n-1$. (26) $\left[z_{n} \rightarrow p\right]_{3}^{0}$. (27) $\left[y_{n}\right]_{3}^{0} \rightarrow[]_{3}^{+} u$.

At step $n^{2}+n+2 k+10, y_{i}, z_{i}(1 \leq i \leq n-1)$ evolve to $y_{i+1}, z_{i+1}, z_{n}$ evolves to $p, \bar{e}_{i, j, 0}(1 \leq i<j \leq n)$ evolve to $\bar{e}_{s(i), s(j), 0}$, where $s(t)=\min (t+1, n+2)$; at same time, $y_{n}$ exits the membrane where it appears, changing the polarization of that membrane to positive.

(28) $\left[\bar{e}_{i, n+1,0} \rightarrow u\right]_{3}^{+}, 1 \leq i \leq n . \quad(29)[p]_{3}^{+} \rightarrow[]_{3}^{0} u$.

In the membranes with label 3 and positive polarization (i.e., the membranes where $y_{n}$ appear in the last step, which means that vertex $v_{n}$ does not belong to the corresponding subset), $\bar{e}_{i, n+1,0}(1 \leq i \leq n)$ evolve to $u$; at the same time, object $p$ exits the membrane, returning the polarization of the membrane to neutral (this makes possible the use of rules of types (22)-(27)).

In the membranes with label 3 and neutral polarization (i.e., the membranes where $y_{n}$ do not appear in the last step, which means that vertex $v_{n}$ belongs to the corresponding subset), using rule of type $(24), \bar{e}_{i, n+1,0}(1 \leq i \leq n)$ evolve to $\bar{e}_{i+1, n+2,0}$ (that is, the edge $\left(v_{i}, v_{n}\right)$ with green color does not belong to $V \times(V-A) \cup(V-A) \times V$, where $A$ is the corresponding subset).

The rules of types (22)-(29) are applied as many times as possible. After $2 n$ steps, a membrane contains an object $\bar{e}_{n+2, n+2,0}$ if and only if that membrane contains a green edge which does not belong to $V \times(V-A) \cup(V-A) \times V$, where $A$ is the corresponding subset. If the object $\bar{e}_{n+2, n+2,0}$ does not appear in a membrane with label 3 (that is, the corresponding subset of vertices is a clique with $k$ vertices and red edges), the object yes will appear, and it eventually exits membrane with label 1 . If the object $\bar{e}_{n+2, n+2,0}$ appears in a membrane with label 3 (that is, the corresponding subset of vertices is not a clique with $k$ vertices and red edges), this membrane is dissolved by the rule (33), release its content to immediately outside membrane with label 2 , and the computation passes to the next stage - checking whether there exists a clique with $l$ vertices and green edges.

(30) $\left[g_{n} \rightarrow g_{n+1} q\right]_{3}^{0}$. (31) $\left[g_{n+1} \rightarrow g_{n+2}\right]_{3}^{0}$.

(33) $\left[\bar{e}_{n+2, n+2,0}\right]_{3}^{-} \rightarrow h . \quad(34)\left[g_{n+3}\right]_{3}^{-} \rightarrow[]_{3}^{-}$yes. (35) $[\mathrm{yes}]_{2}^{0} \rightarrow[]_{2}^{0}$ yes.

(36) $[\mathrm{yes}]_{1}^{0} \rightarrow[]_{1}^{+} u$.

(32) $\left[g_{n+2} \rightarrow g_{n+3}\right]_{3}^{-}$.

At step $n^{2}+3 n+2 k+10$, the object $g_{n}$ evolves to $g_{n+1} q$. At step $n^{2}+3 n+2 k+11$, the object $g_{n+1}$ evolves to $g_{n+2} q$, and object $q$ exits the membrane, changing the polarization to negative (using rule of type (10)). At step $n^{2}+3 n+2 k+12$, 
in the membranes which contain object $\bar{e}_{n+2, n+2,0}, g_{n+2}$ evolves to $g_{n+3}$, and $\bar{e}_{n+2, n+2,0}$ dissolves the membrane with label 3 . In the next step, the next stage of computation starts - checking whether there exists a clique with $l$ vertices and green edges. At step $n^{2}+3 n+2 k+12$, in the membranes which do not contain object $\bar{e}_{n+2, n+2,0}, g_{n+2}$ evolves to $g_{n+3}$, the membranes with label 3 are not dissolved and the polarization remains negative, in the next step they produce the object yes. After two more steps, the object yes changes the polarization of membrane with label 1 to positive, telling us that the associated complete graph with the membrane labeled by 1 has a clique with $k$ vertices and red edges; after that, this membrane with label 1 will not send out objects to membrane with label 0 any more.

(37) $\left[h \rightarrow q q h_{0}\right]_{2}^{0}$.

At step $n^{2}+3 n+2 k+13$, object $h$ evolves to $q q h_{0}$, where $q$ is used to change the polarization, and $h_{0}$ is used to count the cardinality of subsets of vertices.

(38) $[q]_{2}^{0} \rightarrow[]_{2}^{-} u$.

(39) $[q]_{2}^{-} \rightarrow[]_{2}^{0} u$. (40) $\left[\bar{t}_{i} \rightarrow a b\right]_{2}^{-}, 1 \leq i \leq n$.

(41) $\left[\bar{f}_{i} \rightarrow \bar{f}_{i}^{\prime}\right]_{2}^{-}, 1 \leq i \leq n$. (42) $\left[h_{i} \rightarrow h_{i}^{\prime}\right]_{2}^{0}, 0 \leq i \leq l$.

(43) $\left[h_{i}^{\prime} \rightarrow h_{i+1}\right]_{2}^{+}, 0 \leq i \leq l-1$. (44) $[a]_{2}^{0} \rightarrow[]_{2}^{+} u$.

As the rules of type (10)-(17), the rules of type (38)-(45) are used to check whether the cardinality of the corresponding subset of vertices in each elementary membrane with label 2 equals to $l$. Note that the membranes with label 2 containing membrane with label 3 will not evolve anymore, their corresponding subsets of vertices induce cliques with $k$ vertices and red edges.

(46) $\left[h_{l}^{\prime} \rightarrow q q g\right]_{2}^{0}$. (47) $\left[\bar{f}_{i}^{\prime} \rightarrow \bar{y}_{i} \bar{z}_{i}\right]_{2}^{-}, 1 \leq i \leq n . \quad$ (48) $\left[g \rightarrow g_{0}\right]_{2}^{-}$.

(49) $\left[e_{i, j, r} \rightarrow e_{i, j, r-1}\right]_{2}^{\alpha}, 1 \leq i<j \leq n, 1 \leq r \leq 2 l+7, \alpha \in\{+,-, 0\}$.

At step $n^{2}+3 n+2 k+2 l+17$, in the membranes with label 2 and polarization 0 , $h_{l}^{\prime}$ evolves to $q q g$. At step $n^{2}+3 n+2 k+2 l+18$, one copy of $q$ exits the membrane, changing its polarization to negative. In the next step, $\bar{f}_{i}^{\prime}(1 \leq i \leq n)$ evolves to $\bar{y}_{i} \bar{z}_{i}, g$ evolves to $g_{0}, e_{i, j, 0}$ appears for each edge $\left(v_{i}, v_{j}\right)$ with red color, and the other copy of $q$ exits the membrane, changing its polarization to neutral.

(50) $\left[\bar{y}_{i} \rightarrow \bar{y}_{i+1}\right]_{2}^{0}, 1 \leq i \leq n-1 . \quad(51)\left[\bar{z}_{i} \rightarrow \bar{z}_{i+1}\right]_{2}^{0}, 1 \leq i \leq n-1$.

(52) $\left[e_{i, j, 0} \rightarrow e_{s(i), s(j), 0}\right]_{2}^{0}, 1 \leq i<j \leq n+1$, where $s(t)=\min (t+1, n+2)$.

(53) $\left[g_{i} \rightarrow g_{i+1}\right]_{2}^{0}, 1 \leq i \leq n-1$. (54) $\left[\bar{z}_{n} \rightarrow p\right]_{2}^{0}$. (55) $\left[\bar{y}_{n}\right]_{2}^{0} \rightarrow[]_{2}^{+} u$.

At step $n^{2}+3 n+2 k+2 l+20, \bar{y}_{i}, \bar{z}_{i}(1 \leq i \leq n-1)$ evolve to $\bar{y}_{i+1}, \bar{z}_{i+1}, \bar{z}_{n}$ evolves to $p, e_{i, j, 0}(1 \leq i<j \leq n)$ evolve to $e_{s(i), s(j), 0}$, where $s(t)=\min (t+1, n+2)$; at same time, $\bar{y}_{n}$ exits the membrane where it appears, changing the polarization of that membrane to positive.

(56) $\left[e_{i, n+1,0} \rightarrow u\right]_{2}^{+}, 1 \leq i \leq n . \quad(57)[p]_{2}^{+} \rightarrow[]_{2}^{0} u$.

The rules of type (50)-(57) are used to check whether a subset of vertices with $l$ vertices induces a clique with green edges. In $2 n$ steps a membrane contains an object $e_{n+2, n+2,0}$ if and only if that membrane contains a red edge which does not belong to $V \times(V-A) \cup(V-A) \times V$, where $A$ is the corresponding subset. This process finishes after step $n^{2}+5 n+2 k+2 l+19$. 

(58) $\left[g_{n} \rightarrow g_{n+1} q\right]_{2}^{0}$.
(59) $\left[g_{n+1} \rightarrow g_{n+2}\right]_{2}^{0}$.
(60) $\left[g_{n+2} \rightarrow g_{n+3}\right]_{2}^{-}$.
(61) $\left[g_{n+3}\right]_{2}^{-} \rightarrow[]_{2}^{-}$yes.
(62) $[\text { yes }]_{1}^{0} \rightarrow[]_{1}^{+} u$.
(63) $\left[e_{n+2, n+2,0}\right]_{2}^{-} \rightarrow[]_{2}^{+} \mathrm{no}_{1}$.
(64) $\left[\mathrm{no}_{1} \rightarrow \mathrm{no}_{2}\right]_{1}^{0}$.
(65) $\left[\mathrm{no}_{2} \rightarrow \mathrm{no}_{3}\right]_{1}^{0}$.
(66) $\left[\mathrm{no}_{3}\right]_{1}^{0} \rightarrow[]_{1}^{+}$no.

At step $n^{2}+5 n+2 k+2 l+20, g_{n}$ evolves to $g_{n+1} q$. In the next step, $g_{n+1}$ evolves to $g_{n+2} q$, and object $q$ exits the membrane, changing the polarization to negative.

At step $n^{2}+5 n+2 k+2 l+22$, in the membranes with label 2 which do not contain object $e_{n+2, n+2,0}, g_{n+2}$ evolves to $g_{n+3}$, and the polarization remains negative, in the next step they produce the object yes. At step $n^{2}+5 n+2 k+$ $2 l+24$, the object yes exits membrane with label 1 changing the polarization to positive, telling us that the associated complete graph with the membrane with label 1 has a clique with $l$ vertices and green edges.

At step $n^{2}+5 n+2 k+2 l+22$, in the membranes with label 2 which contain object $e_{n+2, n+2,0}, g_{n+2}$ evolves to $g_{n+3}$, and $e_{n+2, n+2,0}$ exit these membranes, changing the polarization of these membranes to positive (the other copies of $e_{n+2, n+2,0}$ cannot exit any more). After two more steps, $\mathrm{no}_{1}$ evolves to $\mathrm{no}_{3}$. At step $n^{2}+5 n+2 k+2 l+25$, if the polarization of a membrane with label 1 is still neutral, then it means that the associated graph with the membrane with label 1 has neither a clique with $k$ vertices and red edges nor a clique with $l$ vertices and green edges. The object $\mathrm{no}_{3}$ exits, evolving to no, also changing the polarization of membrane with label 1 to positive (in order that the objects no remaining in it cannot continue to exit).

(67) $[\mathrm{no}]_{0}^{0} \rightarrow[]_{0}^{+}$no.

(68) $\left[c_{i} \rightarrow c_{i+1}\right]_{0}^{0}, 0 \leq i \leq n^{2}+5 n+2 k+2 l+25$.

(69) $\left[c_{n^{2}+5 n+2 k+2 l+26}\right]_{0}^{0} \rightarrow[]_{0}^{0}$ yes.

If the object no appears in the skin membrane labeled by 0 , then at step $n^{2}+5 n+2 k+2 l+26$, it is send out to the environment, telling us that there exists a complete graph with $n$ vertices and edges colored by red and green that contains neither a clique with $k$ vertices and red edges nor a clique with $l$ vertices and green edges; the computation stops. Note that if there is no object no in the skin membrane, then it means that each complete graph with $n$ vertices and edges colored by red and green contains a clique with $k$ vertices and red edges or a clique with $l$ vertices and green edges, and at step $n^{2}+5 n+2 k+2 l+26$ the polarization of the skin membrane is neutral. The object $c_{n^{2}+5 n+2 k+2 l+25}$ (originating from $c_{0}$ ) evolves to $c_{n^{2}+5 n+2 k+2 l+26}$, and in the next step, $c_{n^{2}+5 n+2 k+2 l+26}$ exits the skin membrane, evolving to the object yes; the computation stops.

From the previous explanation of the use of rules, one can easily see how this $\mathrm{P}$ system works. It is clear that the object yes exits the system if only if the integer $n$ is the value of Ramsey number $R(k, l)$. If $n$ is not the value of $R(k, l)$, then at step $n^{2}+5 n+2 k+2 l+26$ the system sends the object no to the environment.

The formal verification of the above computation is omitted, just pointing out that the construction of the above $\mathrm{P}$ system can be done in polynomial time by a 
deterministic Turing machine. Because the sets of rules associated with the system $\Pi(\langle n, k, l\rangle)$ are recursive, it is enough to note that the amount of necessary resources for defining such system is polynomial with respect to $n$ :

- Size of the alphabet: $(n+2)^{2}(2 n+2 k+2 l+13)+2 n^{2}+n(n-1) / 2+16 n+$ $2 k+2 l+2 \max \{k, l\}+44 \in O\left(n^{3}\right)$

- Initial number of cells: $5 \in O(1)$;

- Initial number of objects: $n(n+1) / 2+2 \in O\left(n^{2}\right)$;

- Number of rules: $n(n-1)(2 n+2 k+6)+n(n+1)(2 l+7)+4 n^{2}+17 n+4 k+4 l+70 \in$ $O\left(n^{3}\right)$

- Maximal length of a rule: $7 \in O(1)$.

\section{Discussion}

Because it is very hard to get the exact values of Ramsey numbers, this is a good way for examining the power of unconventional computing models. In this work, an algorithm is presented in the framework of $\mathrm{P}$ systems to decide whether an integer number $n$ is the value of Ramsey number $R(k, l)$, whose computation time is polynomial with respect to $n$.

Even for small $k$ and $l$, e.g., the case for $R(5,5)$ and $R(6,6)$, it is still very difficult to obtain the values of Ramsey numbers. But "biochemical computers" or "artificial cells" seem very suitable for computing Ramsey numbers. The Ramsey number $R(5,5)$ has the bounds $43 \leq R(5,5) \leq 49[7]$. The value of $R(5,5)$ can be obtained after running our algorithm at most 6 times, one time for each integer from 43 to 48 . In each running of algorithm, the number of biochemical steps is not more than 2591 (computation steps $n^{2}+5 n+2 k+2 l+27 \leq 48^{2}+5 \times 48+2 \times 5+2 \times 5+27=2591$ ).

In general, there are a lower and upper bound of Ramsey number $R(k, l)$

$$
2^{m} \leq R(k, l) \leq\left(\begin{array}{c}
k+l-2 \\
l-1
\end{array}\right)
$$

where $m=\min \{k, l\}$. In order to obtain the exact value of Ramsey number $R(k, l)$, the algorithm has to be run for each integer between the bounds of $R(k, l)$. When $k, l$ are large integers and $k>>l$, the gap between the upper bound and the lower bound can be very big. In these cases, the algorithm given in this work is not efficient enough to obtain the value of Ramsey number $R(k, l)$. It remains open to design a $\mathrm{P}$ system such that the system outputs the value of Ramsey number $R(k, l)$ in a polynomial steps after the integers $k, l$ are inputted into the system.

Although the algorithm presented in this work seems attractive from the theoretical point of view, the following analysis shows that it is not realistic. The algorithm is a brute force one, which tests all possible colorings of $K_{n}$ for a recommended $n$. As shown in the above section, the amount of resources to construct the $\mathrm{P}$ system is $O\left(n^{3}\right)$. But, during the computation, the $\mathrm{P}$ system generates $2^{n(n-1) / 2}$ separate copies of membrane 4 . This cannot happen in this universe for an integer $n$, $43 \leq n \leq 49$. (One can imagine how big such space size is. The human being brain 
has about $2^{33}$ neural cells. The size of $2^{43(43-1) / 2}$ cells is $2^{870}$ times of brain, which is beyond any philosophical limits.) The good time performance in the algorithm is achieved by trading space for time. In general, the brute force algorithm based on trading space for time is not realistic at least in the case of Ramsey numbers. It deserves to investigate how membrane computing leads to some improved algorithms, or new insights into attacking computationally hard problems.

It is worth pointing out that currently, nobody knows how to build a biochemical computer or an "artificial cell" for computing, although it seems as though it might be possible within the biological/chemical principles. Even if no useful biochemical computer is ever built, the research on membrane computing illuminates the problem of simulating biological systems on a classical computer.

The algorithm given in this work is in the framework of $\mathrm{P}$ systems. One can perhaps develop similar polynomial-time algorithms for Ramsey number problem on other massively parallel computation models such as DNA computers, alternating Turing machines and quantum computers. Of course, the real implementation of such algorithms is of great interest and a great challenge.

\section{Acknowledgments}

The work of L. Pan was supported by National Natural Science Foundation of China (Nos. 61033003, 30870826, and 60703047), and Natural Science Foundation of Hubei Province (2008CDB113 and 2008CDB180). The work of the last two authors was supported by Project of Excellence with Investigador de Reconocida Valia, from Junta de Andalucía, grant P08 - TIC 04200 and Project TIN2009-13192 from the Ministerio de Ciencia e Innovación of Spain.

Comments from professor Stanislaw P. Radziszowski and professor Gheorghe Păun are gratefully acknowledged.

\section{References}

[1] P. Erdös, Welcome address, Annals of Discrete Mathematics 28(1985) 1-5.

[2] M.R. Garey, D.J. Johnson, Computers and Intractability. A Guide to the Theory of NP-Completeness (W.H. Freeman, San Francisco, 1979).

[3] Gh. Păun, Computing with membranes, Journal of Computer and System Sciences 61(1)(2000) 108-143.

[4] Gh. Păun, P Systems with active membranes: attacking NP-complete problems, Journal of Automata, Languages and Combinatorics 6(1)(2001) 75-90.

[5] Gh. Păun, Membrane Computing: An Introduction (Springer, Berlin, 2002).

[6] Gh. Păun, G. Rozenberg, A. Salomaa, eds.: Handbook of Membrane Computing (Oxford University Press, 2010).

[7] S.P. Radziszowski, Small Ramsey numbers, Elect. J. Combin., Dynamic Survey 1, revision \#11, August, 2006, http://www.combinatorics.org.

[8] F.P. Ramsey, On a problem of formal logic, Proc. London Math. Soc. 30(1930) 264286.

[9] V. Rosta, Ramsey Theory Applications, Elect. J. Combin., Dynamic Survey 13, December 2004. 